ROSE/TN/2001-05

\title{
DEFECT GENERATION IN CRYSTALLINE SILICON IRRADIATED WITH HIGH ENERGY PARTICLES
}

\author{
M. Kuhnke ${ }^{1, a}$, E. Fretwurst ${ }^{b}$ and G. Lindstroem ${ }^{b}$ \\ ${ }^{a}$ Department of Electronic and Computer Engineering, Brunel University, \\ Howell Building, Middlesex UB8 3PH, United Kingdom \\ ${ }^{b}$ II. Institut für Experimentalphysik, University of Hamburg \\ DESY Bldg. 67b, Luruper Chaussee 149, D-22761 Hamburg, Germany
}

\begin{abstract}
High resistivity silicon with different concentrations of the impurities oxygen and carbon were irradiated with neutrons and charged particles. The DLTS method is used to determine the defect parameters. During irradiation of silicon with particles lattice atoms are displaced and the primary defects silicon interstitials and vacancies form the impurity defects $C_{i}, C_{i} C_{s}, C_{i} O_{i}$ and $V O_{i}$. In the dense displacement regions mainly divacancies $V V$ are formed. The radiation induced defects change the macroscopic parameters of silicon detectors. During irradiation with neutrons mainly clusters are created. During irradiation with charged particles the generation of single isolated displacements is enhanced due to Coulomb scattering. This is main difference between irradiation damage after charged particle and neutron irradiation. The higher radiation tolerance of oxygen enriched silicon after charged particle irradiation is related to the higher introduction rates of impurity defects, because only the reaction kinetic of point defects is influenced by the impurity content. The cluster damage is less particle dependent and the threshold energy at which a recoiled silicon atom starts to create a cluster is estimated to be $300 \mathrm{eV}$.
\end{abstract}

Keywords: Silicon detectors, Radiation damage, Radiation hardness, DLTS PACS: $61.82 . \mathrm{Fk}, 81.40 . \mathrm{Wx}$

\footnotetext{
${ }^{1}$ Corresponding Author: Tel.: +44 1895 203199; E-mail: martin.kuhnke@brunel.ac.uk
} 


\section{Introduction}

In future High Energy Physics (HEP) experiments silicon detectors are used in the inner detector region [1]. Because of the harsh radiation environment the properties of the silicon material are changed and hence the detector parameters as full depletion voltage, leakage current and charge collection efficiency [2]. The results of the studies of the ROSE collaboration of the last few years showed that oxygen enriched silicon is 2-3 times radiation harder than standard FZ silicon after charged hadron irradiation. A simple technique was developed to enriched the silicon wafers on which later the detectors are processed. During a high temperature step oxygen is diffused from a oxide surface layer into the silicon bulk. The maximal oxygen concentrations obtained with this technique are $2-4 \cdot 10^{17} \mathrm{~cm}^{-3}$.

However, the radiation tolerance of oxygen enriched silicon depends on the particle type [3]. No beneficial effect is observed after neutron irradiation. This behaviour is due to the generation of single displacements and regions with a high density of displacements during particle irradiation. Only the defect kinetic of point defects is influenced by the impurity content, e.g. oxygen and carbon.

The Deep Level Transient Spectroscopy (DLTS) method is used to investigate radiation induced defects on the microscopic scale [4]. DLTS is the most powerful and sensitive characterization technique. Activation energies and cross sections of defects which have energy levels in the energy band gap are obtained. The results of this study helped to understand the particle and material dependence of the defect introduction rates after particle irradiation.

\section{Experimental Procedures}

Various high resistivity n-type FZ silicon materials were processed and irradiated with protons of an energy 7-10 MeV, $27 \mathrm{MeV}$ and $23 \mathrm{GeV}, 192 \mathrm{MeV}$ pions $\left(\pi^{+}\right)$and $\mathrm{Be}(\mathrm{d}, \mathrm{n})$ neutrons. The neutron field had a mean energy of $5.3 \mathrm{MeV}$. The impurity content of oxygen and carbon in all investigated samples is several orders of magnitude higher than the doping concentration phosphorus. Simple $\mathrm{p}^{+}-\mathrm{n}-\mathrm{n}^{+}$structures with a guard ring were processed on the wafers. The area of the $\mathrm{p}^{+}$region is $5 \times 5 \mathrm{~mm}^{2}$.

The irradiated diodes were tempered for $80 \mathrm{~min}$ at $60^{\circ} \mathrm{C}$ or $4 \mathrm{~min}$ at $80^{\circ} \mathrm{C}$ to avoid any difference in the annealing state after irradiation. The proportionality of the volume generation current with the fluence is employed to determine the equivalent fluence after high energy particle irradiations. The equivalent fluences for the 7-10 MeV and $27 \mathrm{MeV}$ proton irradiations are calculated from the product of particle fluence and hardness factor. The introduction rates of the various radiation induced defects are calculated from the quotient of the defect concentration and equivalent fluence. The equivalent fluences are in the range from $10^{10} \mathrm{~cm}^{-2}$ to $10^{12} \mathrm{~cm}^{-2}$.

A commercially available DLTS apparatus was employed for defect characterization which is described in more detail elsewhere [5]. The displayed spectra correspond to the sinus correlator function and were obtained with a time window of $200 \mathrm{~ms}$. Also the time windows of $20 \mathrm{~ms}$ and $2 \mathrm{~s}$ and other weighting functions were used. The reverse bias was $10 \mathrm{~V}$ and during filling with electrons a filling pulse $0 \mathrm{~V}$ was applied to the diode. During the filling with holes the forward bias was $-3 \mathrm{~V}$. Both filling pulses had a duration of $100 \mathrm{~ms}$. For the capture measurements the duration of the filling pulses was varied from $10 \mathrm{~ns} / 1 \mu \mathrm{s}$ to $1 \mathrm{~s}$. 


\section{Bulk damage}

The interaction of an impinging particle with the lattice atoms transfers kinetic energy to the atoms and displaces them out of their lattice sites. Such a displaced atom is called Primary Knock on Atom (PKA). The energy spectrum of the produced PKAs depends on the impinging particle type and energy. A PKA loses its kinetic energy by further displacements of lattice atoms and ionization.

A recoiled silicon atom with an energy $\mathrm{E}>10 \mathrm{keV}$ displaces only few silicon atoms at the beginning of its stopping range. Single interstitial-vacancy pairs, referred as Frenkel pairs, are generated along the track. The threshold energy to create a Frenkel pairs is about $20 \mathrm{eV}$. The nuclear stopping power increases strongly, if the recoil energy is $\mathrm{E}<10 \mathrm{keV}$. The stopping range is now less than a few nanometers for the silicon recoils and terminal cluster regions are generated [6]. The collision cascades generated in a silicon crystal by silicon recoils with an energy of $0.4-10 \mathrm{keV}$ are studied in more detail with molecular dynamics (MD) simulation methods [7].

After the primary generation of silicon interstitial $I$ and vacancies $V$ in the crystal lattice a migration of these species at room temperature takes place. It is assumed that the recombination rate of interstitials and vacancies is high in the cluster regions because of the high displacement density. Also the formation of intrinsic defects, mainly the divacancy, occurs in the cluster regions. In the small cluster regions common impurities like oxygen $O_{i}$ and carbon $C_{s}$ have no influence on the defect kinetics, because the mean number of an impurity species in a cluster is less than one. Outside the cluster regions reactions with impurity atoms are possible. A set of reactions is listed below:

V reactions $\quad$ I reactions $\quad C_{i}$ reactions
$V+V \rightarrow V V$
$I+C_{s} \rightarrow C_{i}$
$C_{i}+C_{s} \rightarrow C_{i} C_{s}$
$V+O_{i} \rightarrow V O_{i}$
$C_{i}+O_{i} \rightarrow C_{i} O_{i}$
$\mathrm{V}+\mathrm{VO}_{i} \rightarrow \mathrm{V}_{2} \mathrm{O}$
$C_{i}+V V \rightarrow C_{s}+V$

Thus four types of defects are generated in the high resistivity n-type FZ after low fluence irradiation: the vacancy-interstitial oxygen complex $V O_{i}$, the interstitial carbon-substitional carbon complex $C_{i} C_{s}$, the interstitial carbon-interstitial oxygen complex $C_{i} O_{i}$ and the divacancy $V V$. In the high fluence region also the defect $\mathrm{V}_{2} \mathrm{O}$ is generated. This defect is suggested to be of major importance in defect engineering [8].

\section{Experimental results}

\subsection{Introduction rates}

The annealing of the radiation induced defects during room temperature after the tempering for 80 min at $60^{\circ} \mathrm{C}$ is shown in Fig. 1 . The mobile $C_{i}$ atoms are captured mainly at $C_{s}$ and $O_{i}$ sites. The concentrations of the defects $C_{i} C_{s}$ and $C_{i} O_{i}$ increase. The signal of the transition $V V^{=/-}$is smaller than the signal of the transition $V V^{-/ 0}+$ ?, because only a fraction of the divacancies in the clusters is in the double negative charge state at $\mathrm{T} \approx 125 \mathrm{~K}$. Also the emission parameters 


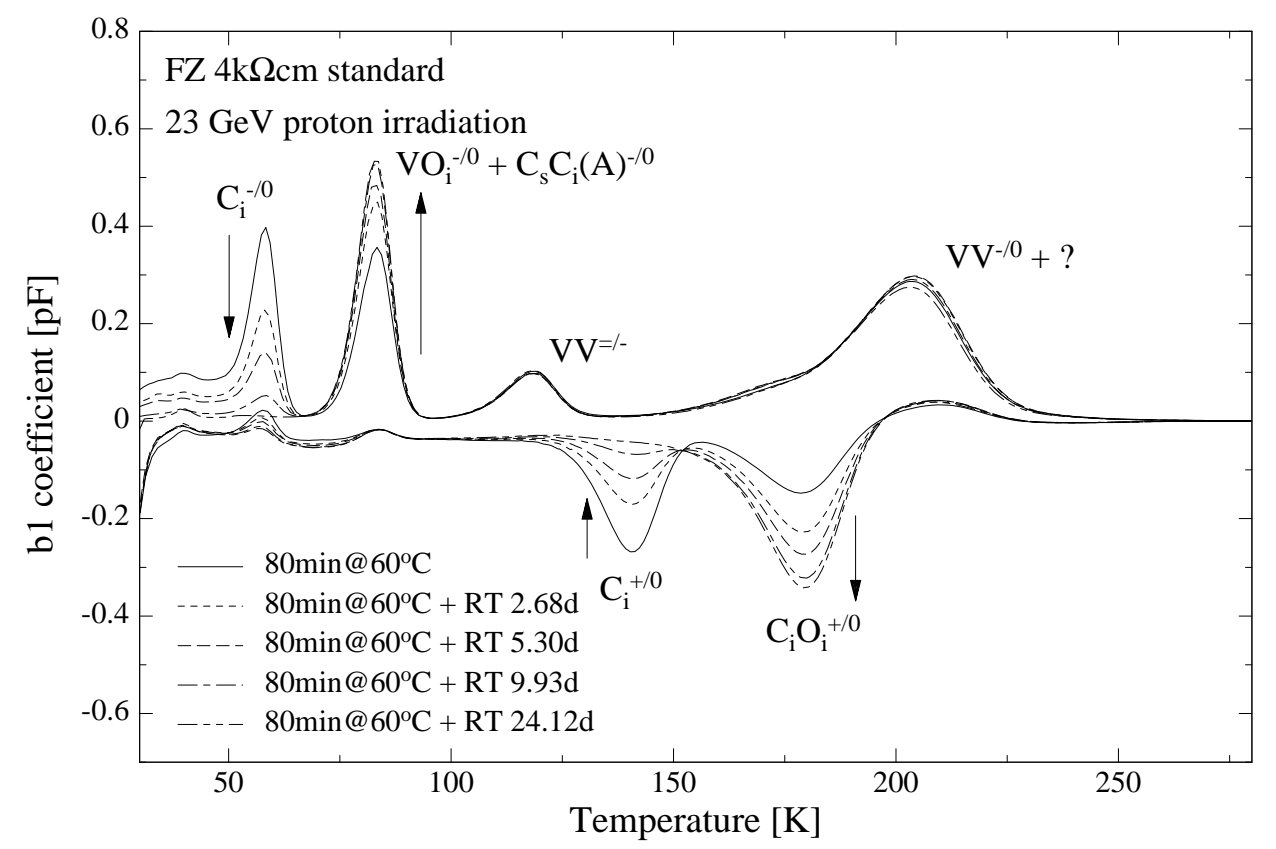

Figure 1: DLTS-spectra of a standard FZ 4k $\Omega \mathrm{cm}$ sample irradiated with $23 \mathrm{GeV}$ protons at different annealing states are shown. After the tempering for $80 \mathrm{~min}$ at $60^{\circ} \mathrm{C}$ the annealing of $C_{i}$ at room temperature is observed. The impurity concentrations are $[\mathrm{O}]=9.0 \cdot 10^{15} \mathrm{~cm}^{-3}$ and $[\mathrm{C}]=6.2$. $10^{15} \mathrm{~cm}^{-3}$.

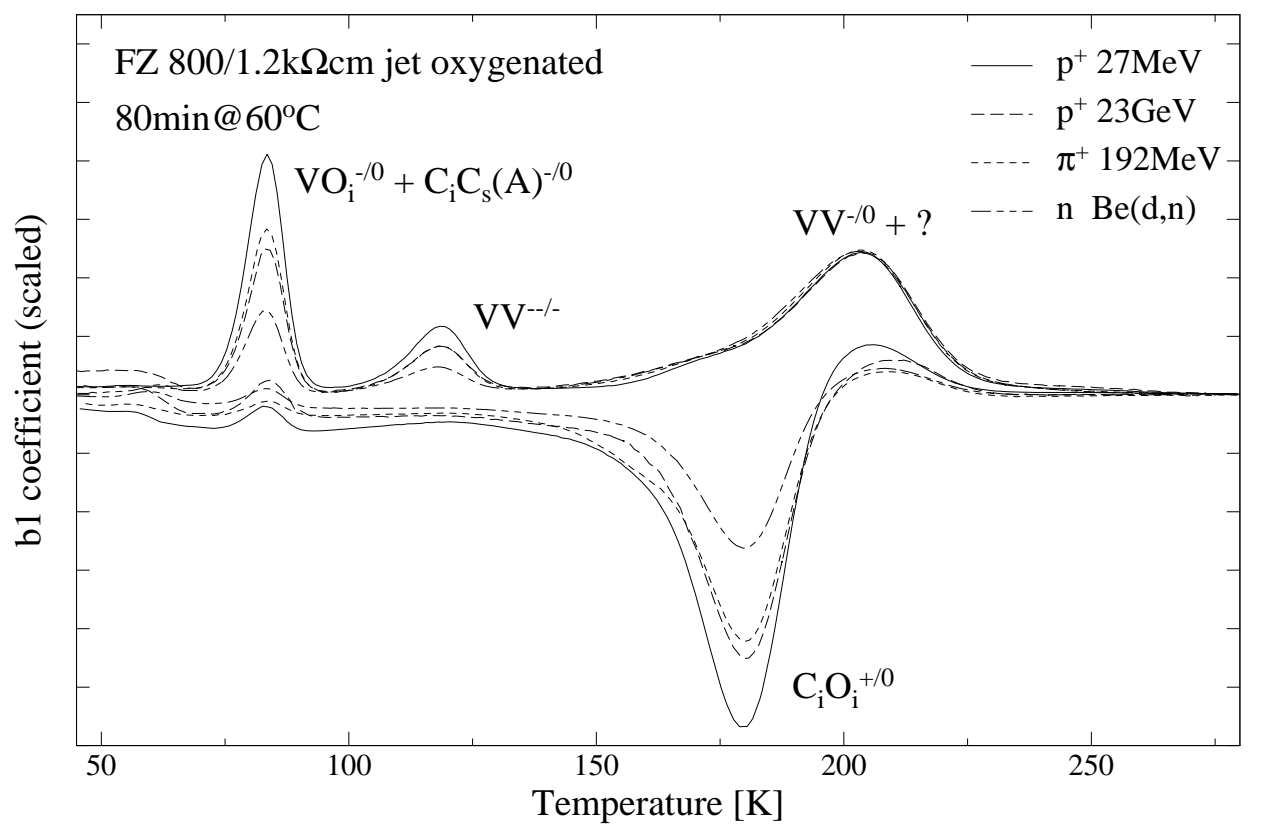

Figure 2: DLTS-spectra of jet-oxygenated FZ 800/1.2k $\Omega \mathrm{cm}$ samples irradiated with different particles are shown. The DLTS-signals are scaled to have a coincidence of the cluster signal $V V^{-/ 0}+$ ?. The impurity concentrations are $[\mathrm{O}]=1.7-2.1 \cdot 10^{17} \mathrm{~cm}^{-3}$ and $[\mathrm{C}]<2.0 \cdot 10^{16} \mathrm{~cm}^{-3}$. 
Table 1: Introduction rates of radiation induced defects for different particles in jet-oxygenated FZ $800 / 1.2 \mathrm{k} \Omega \mathrm{cm}$ samples are listed.

\begin{tabular}{|c|c|c|c|c|c|c|}
\hline $\begin{array}{c}\text { Particle } \\
\text { type }\end{array}$ & $\begin{array}{c}\mathrm{g}\left(V O_{i}\right) \\
{\left[\mathrm{cm}^{-1}\right]}\end{array}$ & $\begin{array}{c}\mathrm{g}\left(C_{i} C_{s}\right) \\
{\left[\mathrm{cm}^{-1}\right]}\end{array}$ & $\begin{array}{c}\mathrm{g}\left(C_{i} O_{i}\right) \\
{\left[\mathrm{cm}^{-1}\right]}\end{array}$ & $\begin{array}{c}\sum g_{\text {point }} \\
{\left[\mathrm{cm}^{-1}\right]}\end{array}$ & $\begin{array}{c}\mathrm{g}\left(V V^{-/ 0}+?\right) \\
{\left[\mathrm{cm}^{-1}\right]}\end{array}$ & $\begin{array}{c}\mathrm{g}\left(V V^{-/=}\right) / \\
\mathrm{g}\left(V V^{-/ 0}+?\right)\end{array}$ \\
\hline$p^{+} 27 \mathrm{MeV}$ & 1.36 & 0.43 & 2.34 & 4.13 & 1.26 & 0.41 \\
\hline$p^{+} 23 \mathrm{GeV}$ & 0.82 & 0.53 & 1.95 & 3.30 & 1.35 & 0.34 \\
\hline$\pi^{+} 192 \mathrm{MeV}$ & 0.67 & 0.67 & 1.83 & 3.17 & 1.30 & 0.30 \\
\hline $\mathrm{n} \mathrm{Be}(\mathrm{d}, \mathrm{n})$ & $\approx 0.10$ & $\approx 0.72$ & 1.14 & 1.96 & 1.38 & 0.22 \\
\hline
\end{tabular}

of the two negative charge states of the divacancy are changed after particle irradiation in comparison to ${ }^{60} \mathrm{Co} \gamma$-photon irradiation during that only single displacements occur. But the emission parameters of the impurity related defects, referred as point defects, are not changed.

Samples of the jet-oxygenated FZ $800 / 1.2 \mathrm{k} \Omega \mathrm{cm}$ were irradiated with neutrons, protons and pions. The DLTS spectra are shown in Fig. 2. The $C_{i}$ annealing is already finished in the oxygen rich FZ silicon. The DLTS spectra are scaled to show the particle independence of the shape of the cluster signal $V V^{-/ 0}+$ ?. The introduction rates are listed in Table 1. Capture measurements are used to separate the defects $V O_{i}$ and $C_{i} C_{s}$. This method works only well for low energetic particle irradiations. The introduction rate of the cluster defect $V V^{-/ 0}+$ ? is nearly particle independent. The ratio $\left[V V^{=/-}\right] /\left[V V^{-/ 0}+?\right]$, which depends on the defect density in the clusters, is particle dependent and has its smallest value after neutron irradiation. The sum of the introduction rates of point defects is higher after charged particle irradiation due to low energetic PKAs produced by Coulomb scattering.

The material dependence of the introduction rates after $23 \mathrm{GeV}$ proton irradiation is given in Table 2. The material with the lowest oxygen concentration is not oxygen enriched. The other materials are oxygenated by high temperature diffusion. The introduction rate of the cluster defect $V V^{-/ 0}+$ ? is independent on the content of oxygen and carbon and also the sum of the introduction rates of the point defects $C_{i} C_{s}, C_{i} O_{i}$ and $V O_{i}$. The annealing of $C_{i}$ was not finished after the tempering for $80 \mathrm{~min}$ at $60^{\circ} \mathrm{C}$ in the sample with the lowest oxygen concentration and an additional storage of about one week at room temperature was needed to accomplish the annealing (see Fig. 1).

Table 2: Introduction rates of radiation induced defects after $23 \mathrm{GeV}$ proton irradiation in various FZ samples with different oxygen and carbon concentrations are listed.

\begin{tabular}{|c|c|c|c|c|c|c|}
\hline$[O]$ & {$[C]$} & $\begin{array}{c}\mathrm{g}\left(V O_{i}\right) \\
{\left[\mathrm{cm}^{-1}\right]}\end{array}$ & $\begin{array}{c}\mathrm{g}\left(C_{i} C_{s}\right) \\
{\left[\mathrm{cm}^{-1}\right]}\end{array}$ & $\begin{array}{c}\mathrm{g}\left(C_{i} O_{i}\right) \\
{\left[\mathrm{cm}^{-1}\right]}\end{array}$ & $\begin{array}{c}\mathrm{g}\left(V V^{-/ 0}+?\right) \\
{\left[\mathrm{cm}^{-1}\right]}\end{array}$ & $\begin{array}{c}\sum g_{\text {point }} \\
{\left[\mathrm{cm}^{-1}\right]}\end{array}$ \\
\hline $10^{16}\left[\mathrm{~cm}^{-3}\right]$ & 0.62 & 1.15 & 1.06 & 1.30 & 1.32 & 3.51 \\
\hline 0.9 & 0.62 & 0.60 & 2.12 & 1.41 & 3.67 \\
\hline 14 & 0.47 & 0.95 & 0.12 & 1.37 & 3.42 \\
\hline 30 & 0.43 & 0.78 & 0.48 & 2.16 & 1.45 & 3.58 \\
\hline 34 & 3.5 & 0.84 & 0.70 & 1.96 & & \\
\hline
\end{tabular}


Table 3: Sum of the introduction rates of the point defects, the introduction rate of the cluster defects and the ratio between both introduction rates for different particles obtained from various samples are listed. The mean values are taken.

\begin{tabular}{|c|c|c|c|}
\hline $\begin{array}{c}\text { Particle } \\
\text { type }\end{array}$ & $\begin{array}{c}\sum g_{\text {point }} \\
{\left[\mathrm{cm}^{-1}\right]}\end{array}$ & $\begin{array}{c}\mathrm{g}\left(V V^{-/ 0}+?\right) \\
{\left[\mathrm{cm}^{-1}\right]}\end{array}$ & $\begin{array}{c}\sum g_{\text {point }} / \\
\mathrm{g}\left(V V^{-/ 0}+?\right)\end{array}$ \\
\hline$p^{+} 7-10 \mathrm{MeV}$ & 6.52 & 1.31 & 3.87 \\
\hline$p^{+} 27 \mathrm{MeV}$ & 4.14 & 1.26 & 3.36 \\
\hline$p^{+} 23 \mathrm{GeV}$ & 3.26 & 1.32 & 2.46 \\
\hline$\pi^{+} 192 \mathrm{MeV}$ & 3.23 & 1.32 & 2.46 \\
\hline $\mathrm{n} \operatorname{Be}(\mathrm{d}, \mathrm{n})$ & 1.82 & 1.30 & 1.43 \\
\hline
\end{tabular}

These results suggest that the generation of the initial dense displacement regions which form later the clusters is particle and material independent and the defect kinetic of the point defects is mainly influenced by the impurities oxygen and carbon. The sum of the introduction rates of the point defects, the introduction rate of the cluster defects and the ratio between both for different particles are listed in Table 3. The listed introduction rates correspond to an annealing state reached after a tempering period for $80 \mathrm{~min}$ at $60^{\circ} \mathrm{C}$. The sum of the introduction rates of the point defects is particle dependent. The introduction rate $\mathrm{g}\left(V V^{-/ 0}+?\right)$ is nearly particle independent.

Four samples of standard FZ $2 \mathrm{k} \Omega \mathrm{cm}$ were irradiated with 7-10 MeV protons and tempered for $4 \mathrm{~min}$ at $80^{\circ} \mathrm{C}$. The particle fluence for all samples is $\Phi_{\text {particle }}=5 \cdot 10^{10} \mathrm{~cm}^{-2}$. The threshold energy at which a recoiled silicon atom starts to create a cluster is estimated from the maximum of the variable $\chi^{2}$ :

$$
\begin{gathered}
\chi^{2}=\frac{\frac{1}{n-2} \sum_{i=1}^{n}\left(g_{i}\left(V V^{-/ 0}+?\right)-\overline{g\left(V V^{-/ 0}+?\right)}\right)^{2}}{\frac{1}{n-1} \sum_{i=1}^{n}\left(g_{i}\left(V V^{-/ 0}+?\right)-g_{0}\left(V V^{-/ 0}+?\right)\right)^{2}} \\
\overline{g\left(V V^{-/ 0}+?\right)}=\frac{1}{n} \sum_{i=1}^{n} g_{i}\left(V V^{-/ 0}+?\right) \\
g_{i}\left(V V^{-/ 0}+?\right)=\frac{\left[V V^{-/ 0}+?\right]_{i}}{\kappa_{i} \cdot \Phi_{\text {particle }, i}}
\end{gathered}
$$

where $n$ is the number of samples and $i$ the index of the samples. The hardness factors $\kappa_{i}$ are calculated for the proton energies 7, 8, 9 and $10 \mathrm{MeV}$. The index $i$ also refers to the proton energy. Only Rutherford scattering is regarded. Also the dependence of the proton energy on the penetration depth is taken into account. The thickness of the silicon layer in which the displacement damage occurs is set equal to the width of the space charge region $\mathrm{W} \approx 70 \mu \mathrm{m}$. The introduction rates $g_{i}\left(V V^{-/ 0}+\right.$ ?) should not depend on the proton energy. The $\chi^{2}$-values for the predicted introduction rate $g_{0}\left(V V^{-/ 0}+?\right)=1.48 \mathrm{~cm}^{-1}$, which corresponds to an annealing state of $4 \mathrm{~min}$ at $80^{\circ} \mathrm{C}$, are calculated for various threshold energies. The results are shown in Fig. 3. The energy $E_{t h} \approx 300 \mathrm{eV}$ obtained from the maximum value of $\chi^{2}$ is proposed to be the threshold energy of cluster generation. 


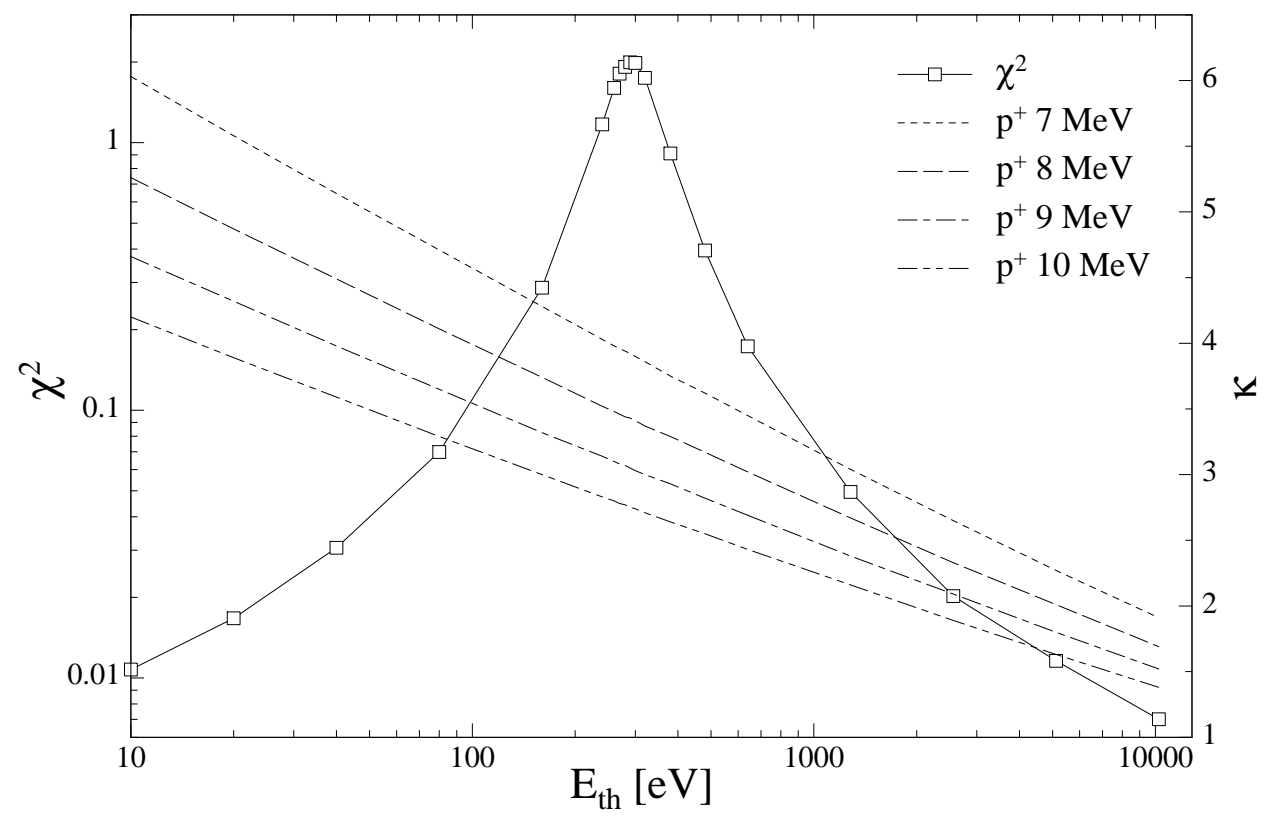

Figure 3: The dependence of the $\chi^{2}$-value and of the hardness factors $\kappa_{i}$ for different proton energies on the threshold energy for cluster generation $E_{t h}$ are shown.

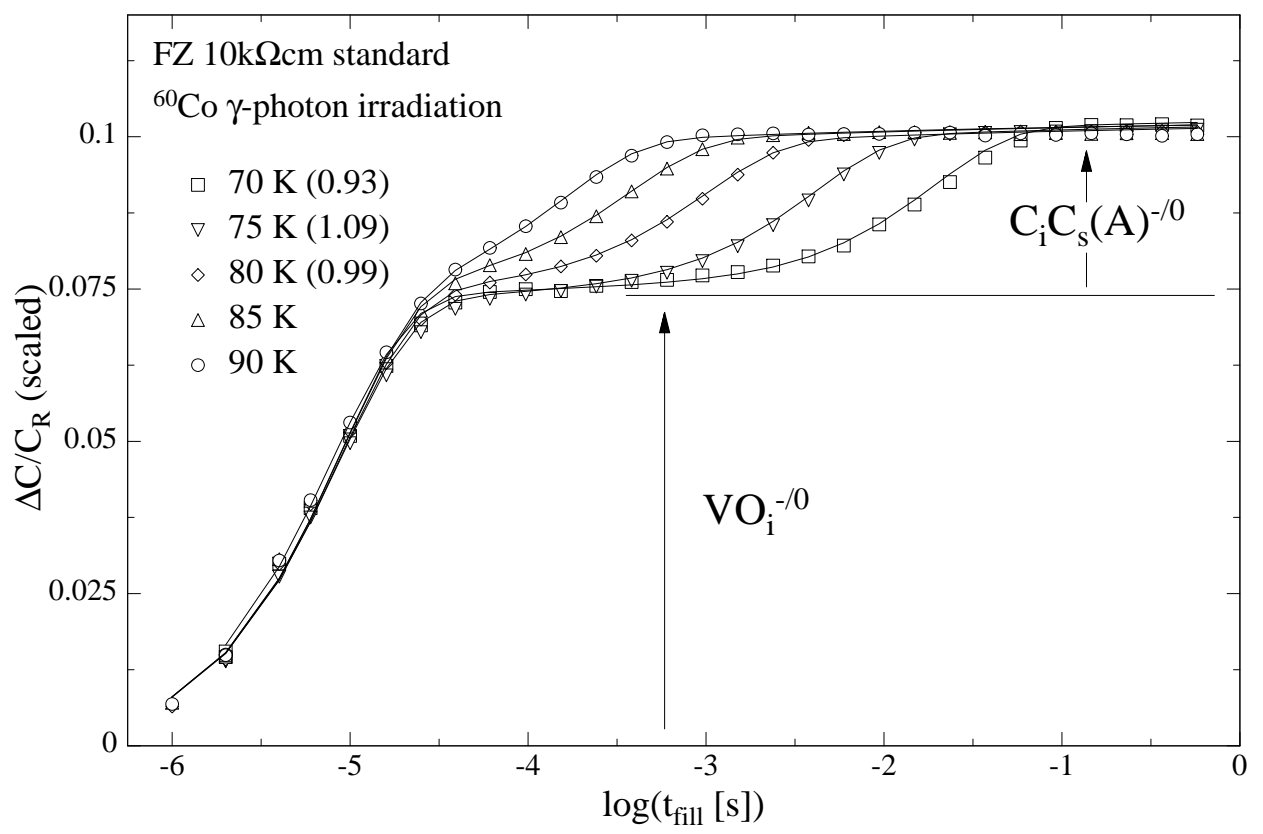

Figure 4: The electrical filling characteristics of the defects $V O_{i}$ and $C_{i} C_{s}$ in a sample of FZ $10 \mathrm{k} \Omega \mathrm{cm}$ after ${ }^{60} \mathrm{Co} \gamma$-photon irradiation at different temperatures are shown (symbols). The signals are scaled with the factors in the parentheses. Also the theoretical predictions of the filling characteristics are shown (lines). The impurity concentrations are $[O]<5.0 \cdot 10^{16} \mathrm{~cm}^{-3}$ and $[C]<0.5 \cdot 10^{16} \mathrm{~cm}^{-3}$. 


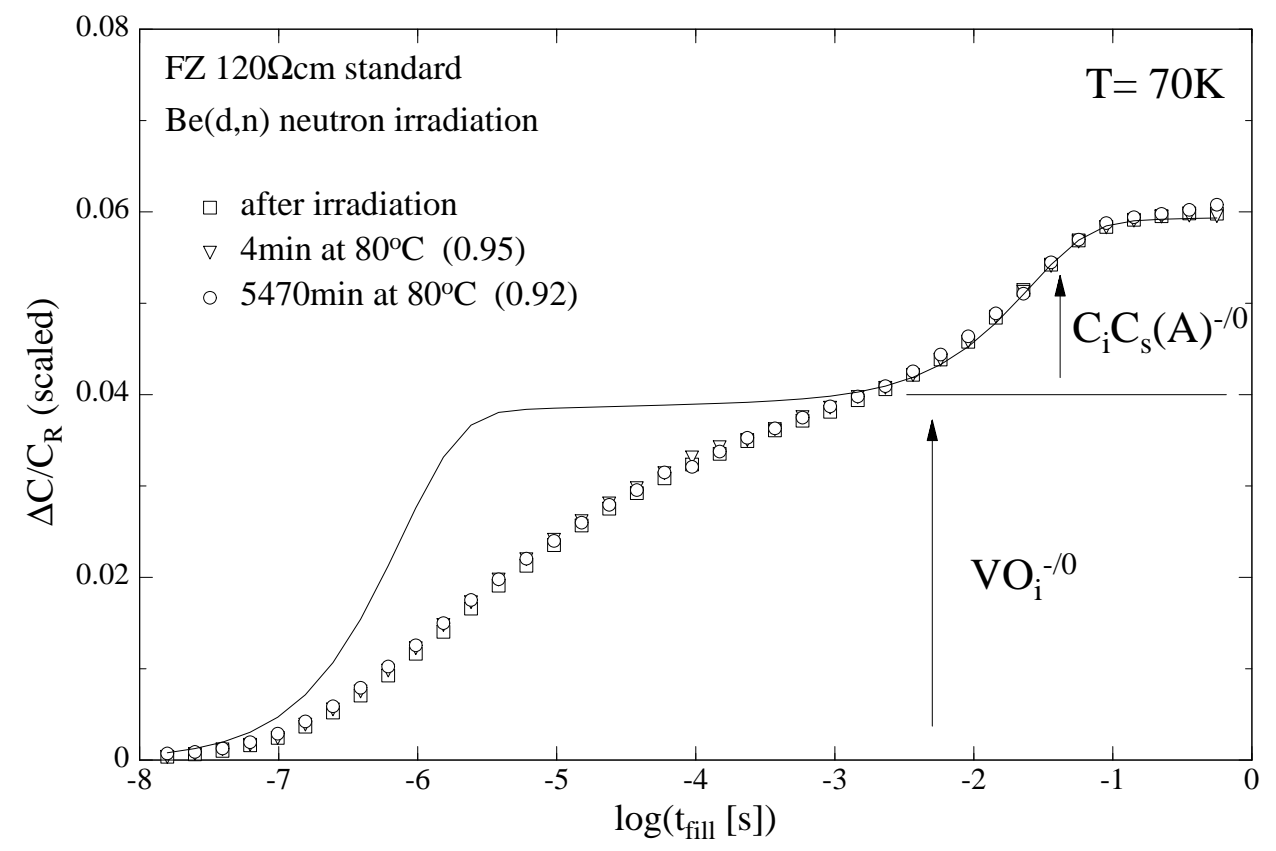

Figure 5: The electrical filling characteristics of the defects $V O_{i}$ and $C_{i} C_{s}$ in a sample of $\mathrm{FZ} 120 \Omega \mathrm{cm}$ after $\operatorname{Be}(\mathrm{d}, \mathrm{n})$ neutron irradiation are shown (symbols). The signals are scaled with the factors in the parentheses. Also the theoretical prediction of the filling characteristic is shown (line). The impurity concentrations are $[O]<5.0 \cdot 10^{16} \mathrm{~cm}^{-3}$ and $[C] \leq 2.0 \cdot 10^{16} \mathrm{~cm}^{-3}$.

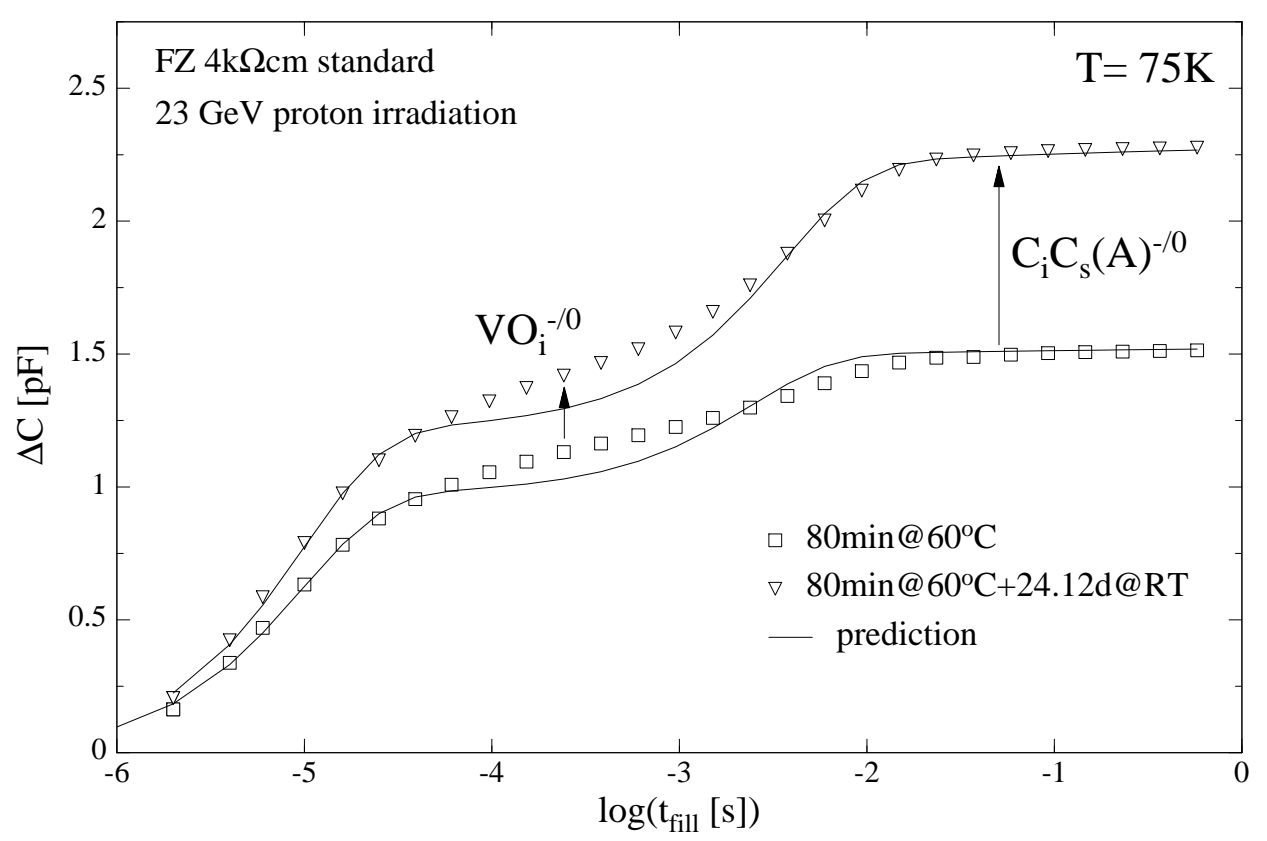

Figure 6: The electrical filling characteristics of the defects $V O_{i}$ and $C_{i} C_{s}$ in a $23 \mathrm{GeV}$ proton irradiated sample of $\mathrm{FZ} 4 \mathrm{k} \Omega \mathrm{cm}$ at two annealing states are shown (symbols). The signal amplitudes of $V O_{i}$ and $C_{i} C_{s}$ increase. Also the theoretical predictions of the filling characteristics are shown (lines). The impurity concentrations are $[O]=9.0 \cdot 10^{15} \mathrm{~cm}^{-3}$ and $[C]=6.2 \cdot 10^{15} \mathrm{~cm}^{-3}$. 


\subsection{Capture measurements}

The electrical filling characteristics $V O_{i}^{0} \rightarrow V O_{i}^{-}$and $C_{i} C_{s}^{0} \rightarrow C_{i} C_{s}^{-}$in a sample of standard FZ $10 \mathrm{k} \Omega \mathrm{cm}$ in the temperature range from $70 \mathrm{~K}$ to $90 \mathrm{~K}$ are shown in Fig. 4. The sample was irradiated with ${ }^{60} \mathrm{Co} \gamma$-photons. No deviation from the theoretical predictions is seen because of sole point defect generation. A separation of the signals of the two defects $V O_{i}$ and $C_{i} C_{s}$ is possible. All states of the defect $V O_{i}$ are already occupied with majority charge carriers for short filling pulses. The capture time constant of the defect $V O_{i}$ is between $10-15 \mu \mathrm{s}$. The configurational switching of the defect $C_{i} C_{s}^{-}(B) \rightarrow C_{i} C_{s}^{-}(A)$ is observed for filling pulses with a longer duration [9].

In the sample of standard FZ $120 \Omega \mathrm{cm}$ irradiated with $\mathrm{Be}(\mathrm{d}, \mathrm{n})$ neutrons the capture rate of the defect $V O_{i}$ is not constant, as shown in Fig. 5. The configurational switching of $C_{i} C_{s}$ is described reasonably by the prediction. The filling characteristics depend not on the annealing state of the radiation damage. It is suggested that the $V O_{i}$ defects are located near the terminal clusters after neutron irradiation and a positive space charge region surrounding the negatively charged cluster core is assumed [10]. Therefore the capture rate of the $V O_{i}$ defects near the terminal clusters is decreased due to the smaller concentration of majority charge carriers.

The electrical filling characteristics $V O_{i}^{0} \rightarrow V O_{i}^{-}$and $C_{i} C_{s}^{0} \rightarrow C_{i} C_{s}^{-}$during annealing are shown in Fig. 6. The sample of standard FZ $4 \mathrm{k} \Omega \mathrm{cm}$ was irradiated with $23 \mathrm{GeV}$ protons and tempered for $80 \mathrm{~min}$ at $60^{\circ} \mathrm{C}$ and then further stored at room temperature. An increase of both signals is observed. This suggest that $C_{i}$ atoms are captured at $V V$ sites at the cluster peripheries. New $C_{s}$ sites are created at the peripheries. The released vacancies are captured at $O_{i}$ sites outside the cluster regions. The measured filling characteristics deviate from the theoretical predictions. The cluster damage produced after particle irradiation is responsible for the change of the configurational switching of $C_{i} C_{s}$ defects located at the cluster peripheries. The configurational switching of $C_{i} C_{s}$ is assumed to be altered by strain and deformation fields originating from the clusters.

\section{Conclusion and Summary}

The following model is proposed to explain the experimental results. For further details see [11]. The primary displacement damage in crystalline silicon is different for various particles. The size of the collision cascade trees and the number of terminal clusters increase with increasing PKA energy. The displacement damage occurs mainly in the cluster regions. It is assumed that the mobility of silicon interstitials is much higher than the mobility of vacancies and hence after the initial annihilation the left interstitials are diluted in the sample volume. The silicon interstitials exchange with substitutional carbon atoms $C_{s}$. The density of vacancies in the clusters is high and the generation of divacancies is favored. No generation of $V O_{i}$ defects occurs in the cluster regions. Single vacancies generated by PKAs with energies less than the threshold energy for cluster generation $E_{t h}=300 \mathrm{eV}$ are more responsible for the generation of $V O_{i}$. Thus the introduction rate of $V O_{i}$ is higher for charged particles due to Coulomb scattering. Only if the PKA energy is greater than $10 \mathrm{keV}$ as for neutron irradiations, the generation of vacancies due to single displacements in the collision cascade regions and perhaps due to escaping from the terminal cluster regions is dominating. The migrating vacancies are captured at the nearest $O_{i}$ sites. Further it is assumed that the migration of $C_{i}$ is influenced by strain and 
deformation fields originating from the clusters. Therefore the $C_{i}$ atoms migrate in direction to the clusters, e.g. the diffusion of liberated interstitial atoms towards the cluster core from a defect-impurity shell was already proposed in [12]. No capture of $C_{i}$ atoms at $O_{i}$ and $C_{s}$ sites occurs on the way to the clusters, because the mean free path length of $C_{i}$ is assumed to be large even in the oxygen and carbon rich silicon. The final migration of $C_{i}$ is constricted in the environment of the clusters. The migration volume decreases with increasing defect density in the clusters, because the strength of the strain and deformation fields increases. The defect density in the clusters is particle dependent. The migrating $C_{i}$ are captured at $O_{i}$ and $C_{s}$ sites in the environment of the clusters. In oxygen and carbon lean silicon migrating $C_{i}$ are also captured at $V V$ sites at the peripheries of the clusters. The released vacancies are captured at $O_{i}$ sites outside the cluster regions. The other migrating $C_{i}$ are captured at the new $C_{s}$ sites. Thus an envelope of $C_{i} C_{s}$ defects around the clusters is created. The configurational switching of $C_{i} C_{s}$ defects in the envelope is changed by strain and deformation fields. Also a positive space charge region surrounding the negatively charged cluster core is assumed. Therefore the capture rate of $V O_{i}$ defects near the clusters is decreased due to the smaller concentration of majority charge carriers.

\section{Acknowledgements}

Support from the European Commission contract no ERBFMRX-CT98-0208 is greatly appreciated.

\section{References}

[1] G. Lindstroem et al. Nucl. Instr. and Meth. A426 (1999) 1

[2] M. Moll Ph.D. thesis, DESY-Report, University of Hamburg (1999)

[3] A. Ruzin et al. IEEE NS-46 (5) (1999) 1310

[4] D.V. Lang J. Appl. Phys. 45 (7) (1974) 3023

[5] Dr. L. Cohausz, PhysTech GmbH, Moosburg

[6] V.A.J. van Lint Nucl. Instr. and Meth. A253 (1987) 453

[7] K. Nordlund et al. Phys. Rev B57 (13) (1998) 7556

[8] B.C. MacEvoy et al. Solid State Phenomena Vols. 57-58 (1997) 221

[9] L.W. Song et al. Phys. Rev B42 (9) (1990) 5765

[10] B.R. Gossik J. Appl. Phys. 30 (8) (1959) 1214

[11] M. Kuhnke Ph.D. thesis, DESY-Report, University of Hamburg (2001)

[12] I.V. Antonova et al. Sov. Phys. Semicond. 23 (6) (1989) 671 